\title{
Variants of Localization and Extension of Hypo- and Aplasia of the Great Saphenous Vein in Patients with Primary Varicose Great Saphenous Veins
}

\author{
Rostyslav Sabadosh
}

\begin{abstract}
The objective of the research was to improve the diagnostics and treatment of patients with primary varicose great saphenous veins by studying their frequency and systematizing the variants of localization and extension of great saphenous vein hypoplasia and aplasia in its trunk.

Materials and Methods. The study included 560 patients with varicose veins of the lower limbs and pathological refluxes in different segments of the great saphenous vein. All the patients underwent triplex ultrasound scan of the lower limb venous system.

Results. Among the patients with pathological reflux in a certain GSV segment, hypo- and aplasia of its segments were observed in $32.5 \%$ of the cases $(95 \% \mathrm{Cl} 28.6-36.6 \%)$. Aplasia of this vein was observed twice as less frequently than hypoplasia $(\mathrm{p}<0.05)$. In $2.3 \%$ of the cases $(95 \% \mathrm{Cl} 1.2-3.9 \%)$, hypoplasia of a certain GSV segment evolved to aplasia, or vice versa. It was found that the GSV trunk may have several hypo- or aplastic regions separated by its normal or varicose segment - bi-level hypo- or aplasia that was observed in $3.8 \%$ of the patients with hypo- or aplasia (95\% Cl 1.6-7.8\%). In bi-level hypo- or aplasia, 2 hypoplastic regions were detected in $85.7 \%$ of the cases $(95 \% \mathrm{Cl} 42.1-99.6 \%)$ and 2 aplastic regions were found in $14.3 \%$ of the cases (95\% Cl 0.4-57.9\%). There were proposed to distinguish the following variants of GSV hypo- and aplasia: 1 ) simple: total, proximal, segmental and distal; 2) bi-level: proximal segmental, distal segmental and bi-segmental. In addition, for every dysplastic vein segment, the type of malformation should be indicated, namely hypoplasia, aplasia, or hypo/aplasia.

Conclusions. The study conducted allowed assessing the relationship between the variants of GSV hypo- and aplastic segment localization and extension and different variations of pathological refluxes of the GSV in its trunk for further choice of surgical tactics.
\end{abstract}

\section{Keywords}

varicose veins; great saphenous vein; hypoplasia; aplasia; classification.

Ivano-Frankivsk National Medical University, Ivano-Frankivsk, Ukraine

Corresponding author: r.sabadosh@gmail.com

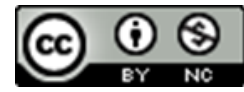

Copyright (C)Rostyslav Sabadosh, 2019

\section{Problem statement and analysis of the latest research}

According to the theory of the angioguiding nerves, the formation of the venous anatomy of the lower limbs is induced by three embryonic nerves, namely the sciatic (axial) nerve running along the central axis of the limb, the ventrally located pre-axial (or femoral) nerve and the dorsally located post-axial nerve that later becomes the posterior cutaneous nerve of the thigh [1]. The axial venous plexus becomes the sural nerve and the trunk of the small saphenous vein (SSV), the post-axial plexus forms its cranial extension, and the pre-axial plexus gives rise to the femoral vein and the great saphenous vein 


\section{Variants of Localization and Extension of Hypo- and Aplasia of the Great Saphenous Vein in Patients with Primary Varicose Great Saphenous Veins - 2/11}

(GSV). There are venous malformations depending on the abnormal development of the axial, pre-axial and post-axial venous plexus.

The venous malformations of the superficial venous system are mainly caused by an abnormal development of the pre-axial and post-axial plexuses. The venous malformations of the post-axial plexus involve the SSV, while the venous malformations of the pre-axial plexus include: 1) (complete or partial) GSV trunk duplication; 2) aplasia or hypoplasia of the GSV segments; 3) isolated anterior accessory GSV reflux in the common femoral vein [2]. The most common venous malformations of the superficial venous system are aplasia or hypoplasia of the GSV that affect $12-39.9 \%$ of the general population $[3,4,5]$.

According to the international terminology in modern phlebology, aplasia and hypoplasia are defined as the incomplete development of a vein or a segment of a vein. In aplasia, in contrast to agenesis, the vein is present; however, it is diminutive in size and its structure is like that in the embryo. It is impossible to detect the vein when using ultrasound scan. In hypoplasia, the vein is visualized during ultrasound scan; however, its caliber is $\leq 50 \%$ of normal values [6]. GSV hypoplasia is diagnosed when its caliber is $<2.5 \mathrm{~mm}$ [7].

Hypoplasia and aplasia of the GSV are most commonly unilateral [8] and affect both the right limb and the left limb equally [9]. Most of them are segmental involving one segment of the GSV only. In this case, the blood flows through the suprafascial branch of the GSV (the superficial, anterior or posterior accessory GSV) that connects the start and end points of its hypoplastic or aplastic segments. However, there was evidence for hypoplasia of the GSV extending to the inguinal area [10]. The incidence rate of such hypoplasia localization is $2.4 \%[3]$.

In most cases, hypoplasia and aplasia of the GSV are found in the upper calf or the lower thigh $(92.8 \%)$ [8]. However, other localizations are possible.

Hypo- and aplasia of the GSV cause no symptoms and are not visible. At the same time, there is convincing evidence that the patients with vari- cose veins develop hypo- and aplasia of the GSV more often than those without this pathology [14]. The individuals with hypo- and aplasia of the GSV develop varicose veins more often since the superficial vein that connects the start and end points of its hypoplastic or aplastic segments (the superficial, anterior or posterior accessory GSV) has much thinner wall and a weaker muscular layer in comparison with the GSV and is surrounded exclusively by subcutaneous fatty tissue without supporting structures such as the saphenous fascia, that can prevent its dilation $[4,5]$.

Hypoplasia and aplasia of the GSV increase the risk of varicose GSV, as well as contribute to changes in the pathways of pathological refluxes. Therefore, the effect of these dysplastic changes on the development and progression of varicose veins is undeniable. Despite this, a number of important issues regarding the role of hypoplasia and aplasia of the GSV in the development of this disease remain unresolved; a comprehensive and simple systematization of the variants of localization and extension of GSV hypoplasia and aplasia and their relationship with various variants of pathological reflux spread in case of varicose veins for the choice of therapeutic tactics are among them.

The objective of the research was to improve the diagnostics and treatment of patients with primary varicose GSV by studying their frequency and systematizing the variants of localization and extension of GSV hypo- and aplasia in its trunk.

\section{Materials and Methods}

The study included 560 patients with varicose veins of the lower limbs. There were $403(72.0 \%)$ females and $157(28.0 \%)$ males. The patients' age ranged from 18 to 83 years. The average patients' age was 49.6 years.

The presence of varicose veins $\left(\mathrm{C}_{2}-\mathrm{C}_{6}\right.$ clinical classes according to the Clinical Etiology Anatomy Pathophysiology (CEAP) classification [15]) with pathological refluxes in any GSV segment served as a criterion for inclusion of patients in the study. The saphenofemoral reflux was diagnosed in 527 $(94.1 \%)$ patients; in $33(5.9 \%)$ patients, reflux was 


\section{Variants of Localization and Extension of Hypo- and Aplasia of the Great Saphenous Vein in \\ Patients with Primary Varicose Great Saphenous Veins - 3/11}

localized only in the larger or smaller GSV segment without involving the area of its origin. Exclusion criteria included the presence of post-thrombotic syndrome, venous or arteriovenous angiodysplasias and acquired arteriovenous fistulas.

According to the CEAP $\mathrm{C}$ classification, the structure of the limbs was as follows: $\mathrm{C}_{2}$ clinical class $-75(13.4 \%)$ limbs; $\mathrm{C}_{3}$ clinical class $254(45.4 \%)$ limbs; $\mathrm{C}_{4 \mathrm{a}}$ clinical class - $90(16.1 \%)$ limbs; $\mathrm{C}_{4 \mathrm{~b}}$ clinical class $-87(15.4 \%)$ limbs; $\mathrm{C}_{5}$ clinical class $-39(7.0 \%)$ limbs; $\mathrm{C}_{6}$ clinical class $15(2.7 \%)$ limbs.

All the patients underwent triplex ultrasound scan of the lower limb venous system by means of the GE Healthcare LOGIQ e ultrasound machine (GE Healthcare, UK) and TOSHIBA ffa-580a/f7 ultrasound machine (TOSHIBA, Japan) using linear transducers with a frequency range of 4.0 10.0 $\mathrm{MHz}$ and convex transducers with a frequency range of 2.0 - $5.5 \mathrm{MHz}$. The survey methodology recommended by the international consensus documents was used $[16,17]$.

The study was carried out in compliance with the World Medical Association (WMA) Declaration of Helsinki Ethical Principles for Medical Research Involving Humans (1974), adapted at the $41^{\text {st }}$ WMA General Assembly in Hong Kong (1989) and Ethical Principles and Guidelines for the Protection of Human Subjects of Research - the Belmont Report (April 18, 1979). The compliance with bioethical standards in a scientific study was confirmed by the expert opinion of the Ethics Committee in the Ivano-Frankivsk National Medical University.

For statistical data analysis, a database was created in Microsoft Excel 2013 (Microsoft, USA), installed due to cloud-based subscription of our university to Office 365 . For statistical computing, a free software environment $\mathrm{R}$ (R Foundation for Statistical Computing, Vienna, Austria) was used [18].

The frequency of qualitative indicators was presented in absolute frequencies ( $\mathrm{n}$ ) and relative frequencies (\%), indicating the $95 \%$ confidence interval (CI) as "n (95\% CI)" if necessary. The exact $\mathrm{CI}$ was calculated using the $\mathrm{R}$ software. The comparison of two groups by a qualitative binary indicator was carried out using the Fisher's exact test. When testing statistical hypotheses, the critical significance level $(\mathrm{p})$ equaled 0.05 .

\section{Results}

Out of 560 limbs with varicose GSV, 182 limbs developed hypo- and aplasia of the GSV segments, namely in $32.5 \%$ of the cases (95\% CI 28.6-36.6\%): hypoplasia was observed in 114 (20.4\%; 95\% CI $17.1-23.9 \%$ ) cases; aplasia was found in 55 (9.8\%; 95\% CI 7.5-12.6\%) cases; hypoplasia of one segment alongside with aplasia of other segment (hypoplasia/aplasia) were detected in 13 (2.3\%; 95\% CI 1.2-3.9\%) cases.

Hypo- or aplasia of the whole GSV segment (from $5 \mathrm{~cm}$ to along its entire length) was detected in $175(96.2 \%)$ out of 182 limbs, while hypo- or aplasia of two segments separated by a healthy or varicose section of GSV was detected in 7 (3.8\%) cases only. Out of 175 limbs with hypo- and/or aplasia of the whole GSV trunk segment, 109 (61.7\%; 95\% CI 54.1-68.9\%) limbs developed only hypoplasia of this segment; 54 (30.9\%; 95\% CI 24.1-38.3\%) limbs developed only aplasia that was significantly less frequently $(\mathrm{p}<0.001) ; 13(7.4 \%$; 95\% CI 4.0$12.4 \%$ ) limbs developed hypoplasia of a certain part of this segment and aplasia of its other part (significantly less frequently than both hypoplasia and aplasia; both $\mathrm{p}<0.001$ ).

The variants of hypo- and aplasia of the GSV we detected did not fit into any classification we knew; therefore, we developed an own systematization of these variants.

In 23 (21.3\%; 95\% CI 14.0-30.2\%) out of 108 limbs with hypoplasia of one GSV segment, it extended from the origin of this vein near the medial malleolus to a certain level, but not proximally to the pre-terminal valve, namely: in 4 cases, it extended to the level of the knee bend; in 4 cases, hypoplasia extended to the junction of the lower and middle thirds of the thigh; in 4 cases, it extended to the junction of the middle and upper thirds of the thigh; in 9 cases, hypoplasia extended to the pre-terminal valve without affecting only the area of the GSV origin. Such variant of localization was defined as distal hypoplasia (Fig. 1d). 

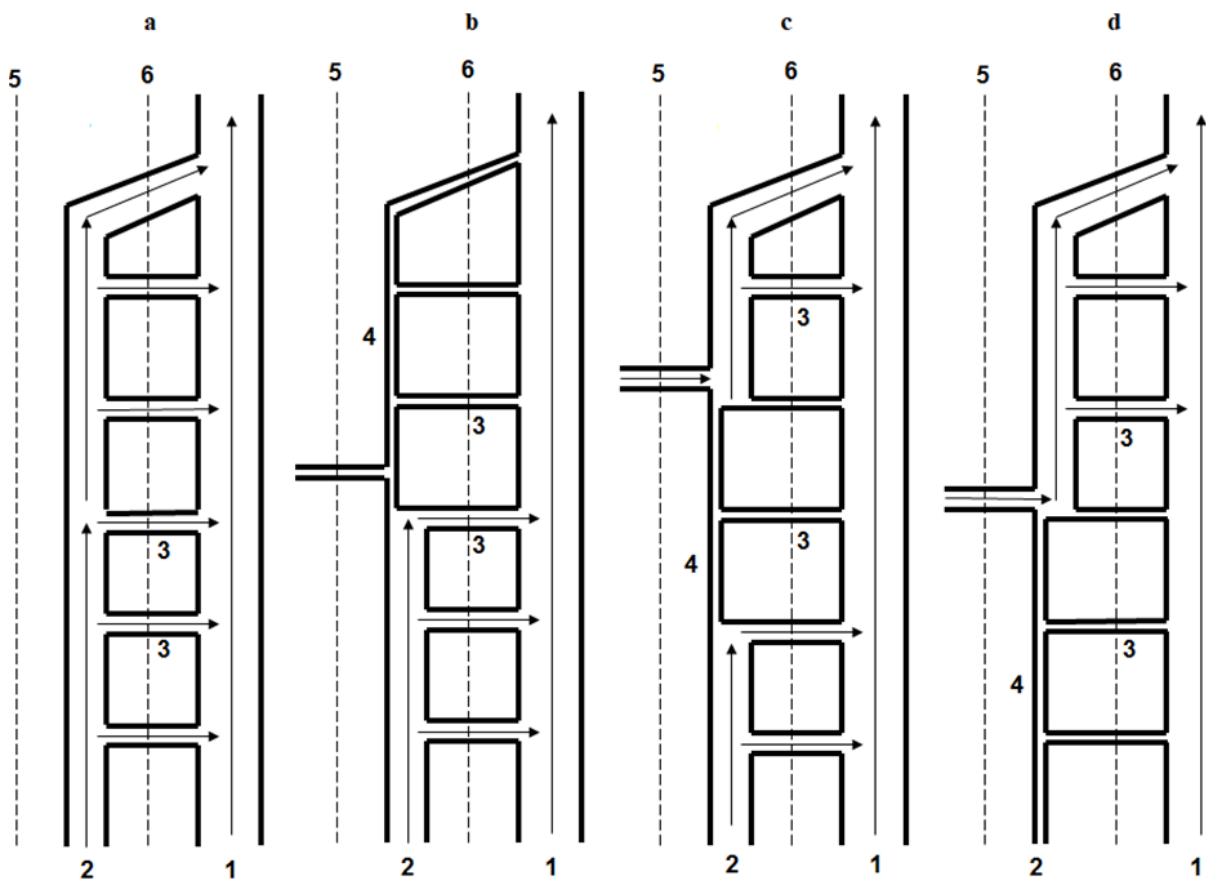

Figure 1. Schematic presentation of the variants of hypoplastic GSV segment localization in the presence of 1 hypoplastic segment (the arrows indicate the direction of blood flow): a) norm; b) proximal hypoplasia; c) segmental hypoplasia; d) distal hypoplasia. 1 - deep vein; 2 - GSV; 3 - perforating veins; 4 hypoplastic GSV region; 5 - projection of the saphenous fascia; 6 - projection of the muscular fascia.

Table 1. Localization and extension of segmental GSV hypoplasia in varicose veins of the lower limbs.

\begin{tabular}{l|c}
\hline Localization of segmental GSV hypoplasia & Absolute detection frequency $(\% ; 95 \% \mathrm{CI})$ \\
\hline Middle third of the tibia only & $10(12.0 ; 5.9-21.0)$ \\
Middle and upper thirds of the tibia & $9(10,8 ; 5.1-19.6)$ \\
Middle third of the tibia - lower third of the thigh & $7(8.4 ; 3.5-16.6)$ \\
Middle third of the tibia - middle third of the thigh & $14(16.9 ; 9.5-26.7)$ \\
Middle third of the tibia - upper third of the thigh & $1(1.2 ; 0.0-6.5)$ \\
Upper third of the tibia only & $11(13.3 ; 6.8-22.5)$ \\
Upper third of the tibia and lower third of the thigh & $11(13.3 ; 6.8-22.5)$ \\
Upper third of the tibia - middle third of the thigh & $12(14.5 ; 7.7-23.9)$ \\
Upper third of the tibia - upper third of the thigh & $1(1.2 ; 0.0-6.5)$ \\
Lower third of the thigh only & $2(2.4 ; 0.3-8.4)$ \\
Lower and middle thirds of the thigh & $5(6.0 ; 2.0-13.5)$ \\
Middle third of the thigh only & $0(0.0 ; 0.0-4.3)$ \\
\hline Total & 83 \\
\hline
\end{tabular}

In 2 (1.9\%; 95\% CI 0.2-6.5\%) other limbs, hypoplasia extended from a certain level of the GSV up to the terminal valve without involving the area of the GSV origin: in 1 case, it extended from the level of the knee bend, while in the other case, it extended from the junction of the lower and middle 

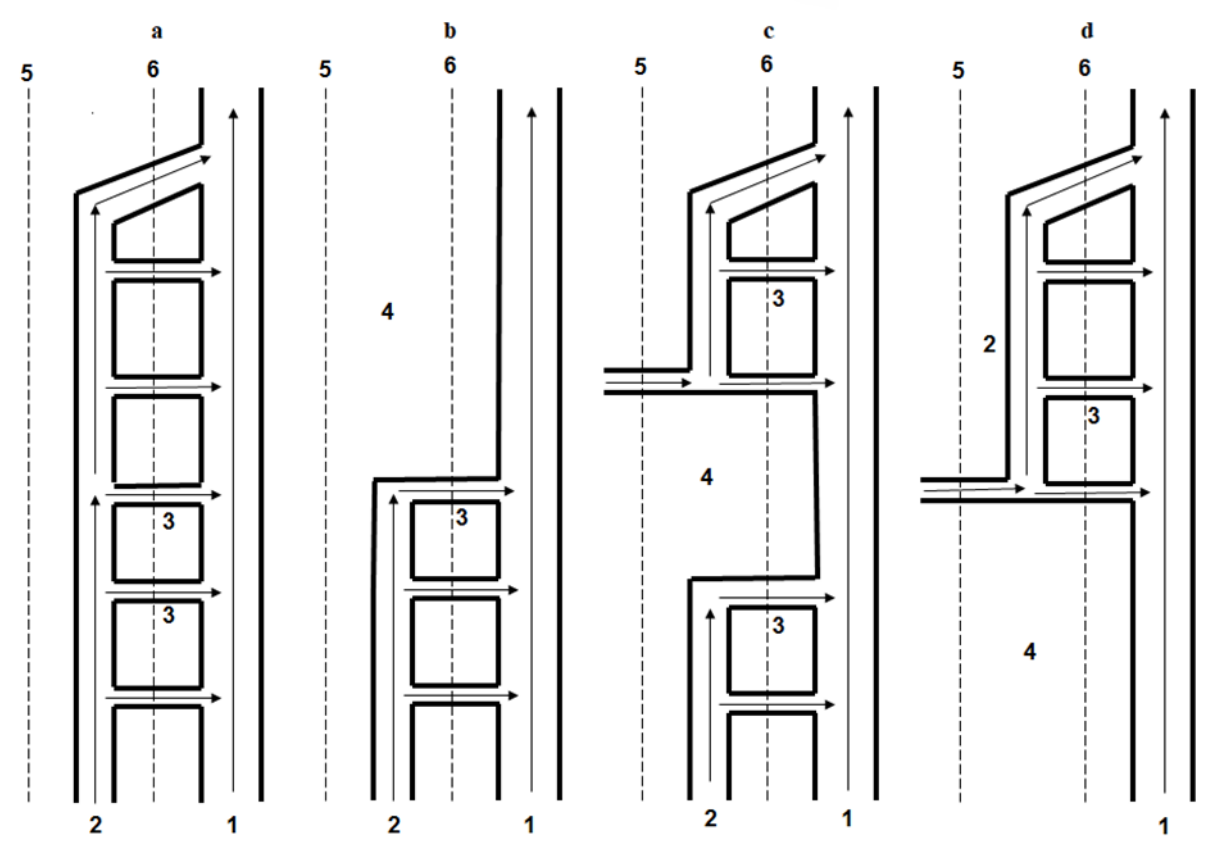

Figure 2. Schematic presentation of the variants of aplastic GSV segment localization in the presence of 1 aplastic segment (the arrows indicate the direction of blood flow): a) norm; b) proximal aplasia; c) segmental aplasia; d) distal aplasia. 1 - deep vein; 2 - GSV; 3 - perforating veins; 4 - aplastic region of the saphenous vein; 5 - projection of the saphenous fascia; 6 - projection of the muscular fascia.

Table 2. Localization and extension of segmental GSV aplasia in varicose veins of the lower limbs.

\begin{tabular}{l|c}
\hline Localization of segmental GSV aplasia & Absolute detection frequency $(\% ; 95 \% \mathrm{CI})$ \\
\hline Middle third of the tibia only & $5(9.4 ; 3.1-20.7)$ \\
Middle and upper thirds of the tibia & $3(5.7 ; 1.2-15.7)$ \\
Middle third of the tibia - lower third of the thigh & $7(13.2 ; 5.5-25.3)$ \\
Middle third of the tibia - middle third of the thigh & $9(17.0 ; 8.1-29.8)$ \\
Middle third of the tibia - upper third of the thigh & $0(0.0 ; 0.0-6.7)$ \\
Upper third of the tibia only & $5(9.4 ; 3.1-20.7)$ \\
Upper third of the tibia and lower third of the thigh & $5(9.4 ; 3.1-20.7)$ \\
Upper third of the tibia - middle third of the thigh & $10(18.9 ; 9.4-32.0)$ \\
Upper third of the tibia - upper third of the thigh & $2(3.8 ; 0.5-13.0)$ \\
Lower third of the thigh only & $1(1.9 ; 0.0-10.1)$ \\
Lower and middle thirds of the thigh & $5(9.4 ; 3.1-20.7)$ \\
Middle third of the thigh only & $1(1.9 ; 0.0-10.1)$ \\
\hline Total & 53 \\
\hline
\end{tabular}

thirds of the thigh. Such variant of localization was defined as proximal hypoplasia (Fig. 1b).

In other 83 (76.8\%; 95\% CI 67.8-84.4\%) cases, hypoplastic segment of the GSV was found in its middle part without reaching either the saphenofemoral junction or the medial malleolus. This 


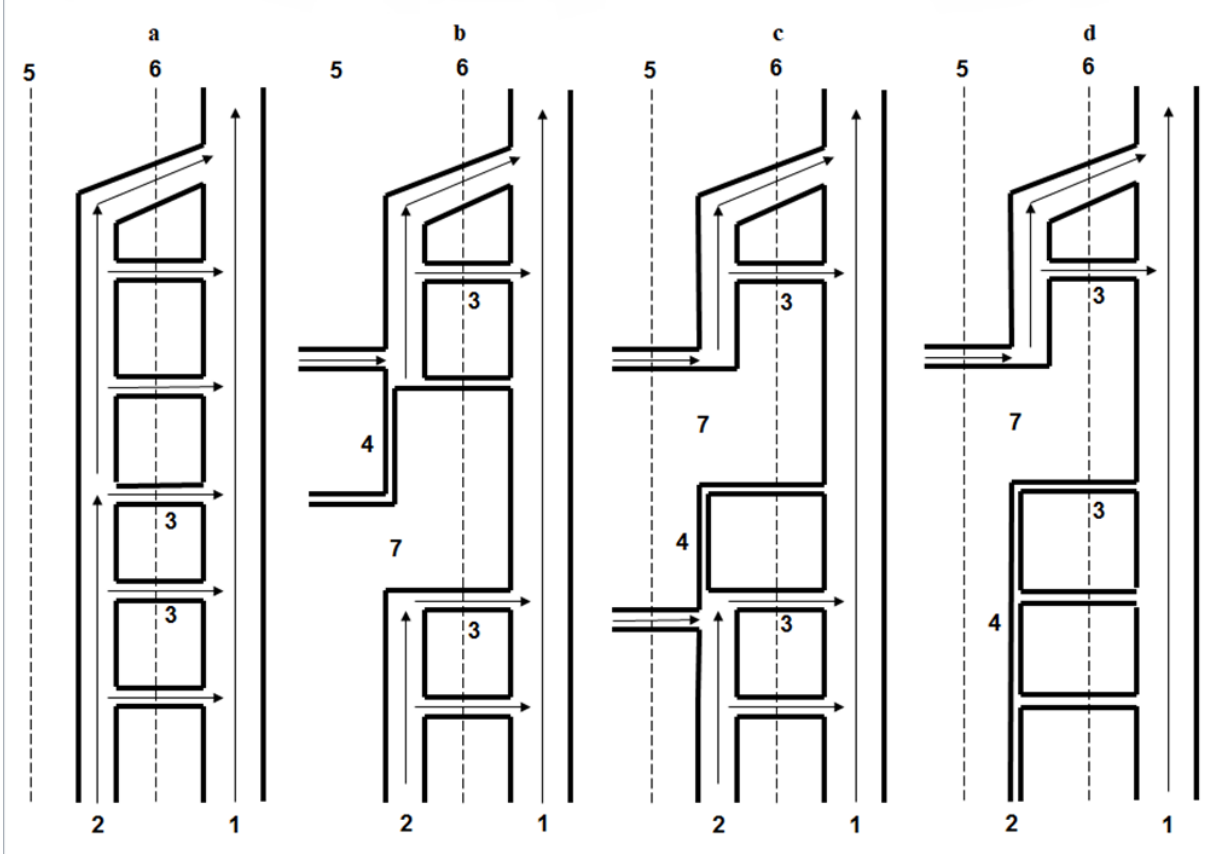

Figure 3. Schematic presentation of the variants of hypo-/aplastic GSV segment localization in the presence of 1 hypo-/aplastic segment (the arrows indicate the direction of blood flow): a) norm; b) segmental hypo-/aplasia with more distal location of aplasia; c) segmental hypo-/aplasia with more distal location of hypoplasia; d) distal hypo-/aplasia with more distal location of hypoplasia. 1 - deep vein; 2 -

GSV; 3 - perforating veins; 4 - hypoplastic GSV region; 5 - projection of the saphenous fascia; 6 projection of the muscular fascia; 7 - aplastic GSV region.

variant of localization was defined as segmental hypoplasia (Fig. 1c). The localization and extension of such hypoplasia were different (Table 1).

In aplasia of one segment of the GSV trunk (54 limbs), in contrast to hypoplasia, there was observed no case of its proximal localization (Fig. 2b) and distal aplasia was found in $1(1.9 \% ; 95 \% \mathrm{CI}$ $0.0-9.9 \%$ ) case (Fig. $2 \mathrm{~d}$ ): the GSV trunk was not visualized in the lower and middle thirds of the tibia.

In other 53 (98.1\%; 95\% CI 90.1-100.0\%) limbs, aplasia was segmental (Fig. 2c). Its extension differed among the patients (Table 2).

Only in $1(7.7 \%$; $95 \%$ CI $0.2-36.0 \%)$ out of 13 limbs with hypoplasia of a certain part of the GSV segment and aplasia of its other part, there was observed distal hypo-/aplasia when the whole tibial segment of the vein was hypoplastic and the vein was not visualized in the lower third of the thigh (Fig. 3d). In other 12 (92.3\%; 95\% CI 64.0-99.8\%) cases, hypo-/aplasia was segmental with more proximal location of hypo- or aplasia (Fig. 3b,c). The following variants of segmental hypo-/aplasia localization and extension were observed (Table 3).

There was no case of hypo- or aplasia of the entire GSV.

In $1(14.3 \%)$ out of 7 cases of two individual segments of hypo- and/or aplasia of the GSV separated by its healthy or varicose section, only aplasia was detected. In this patient, the GSV was not visualized in the middle and upper thirds of the tibia and the middle third of the thigh. Thus, healthy or varicose GSV sections were detected between the aplastic segments, as well as more proximally and distally to them. Such variant of aplasia was defined as bi-segmental aplasia (Fig. 4a). 


\section{Variants of Localization and Extension of Hypo- and Aplasia of the Great Saphenous Vein in \\ Patients with Primary Varicose Great Saphenous Veins - 7/11}

Table 3. Localization and extension of segmental GSV hypo-/aplasia in varicose veins of the lower limbs.

Localization of segmental GSV hypo-/aplasia

Aplasia in the middle third of the tibia and hypoplasia in its upper third

Hypoplasia in the middle third of the tibia and aplasia in its upper third

Aplasia in the middle third of the tibia and hypoplasia in its upper third and the lower third of the thigh

Aplasia in the middle and upper thirds of the tibia and hypoplasia in the lower third of the thigh

Hypoplasia in the middle third of the tibia and aplasia in its upper third and the lower third of the thigh

Hypoplasia in the middle and upper thirds of the tibia and aplasia in the lower and middle thirds of the thigh

Hypoplasia in the middle third of the tibia and aplasia in its upper third and the lower and middle thirds of the thigh

Aplasia in the upper third of the tibia and hypoplasia in the lower third of the thigh

Hypoplasia in the upper third of the tibia and aplasia in the lower third of the thigh

Aplasia in the upper third of the tibia and hypoplasia in the lower and middle thirds of the thigh

Total

Absolute

detection

frequency
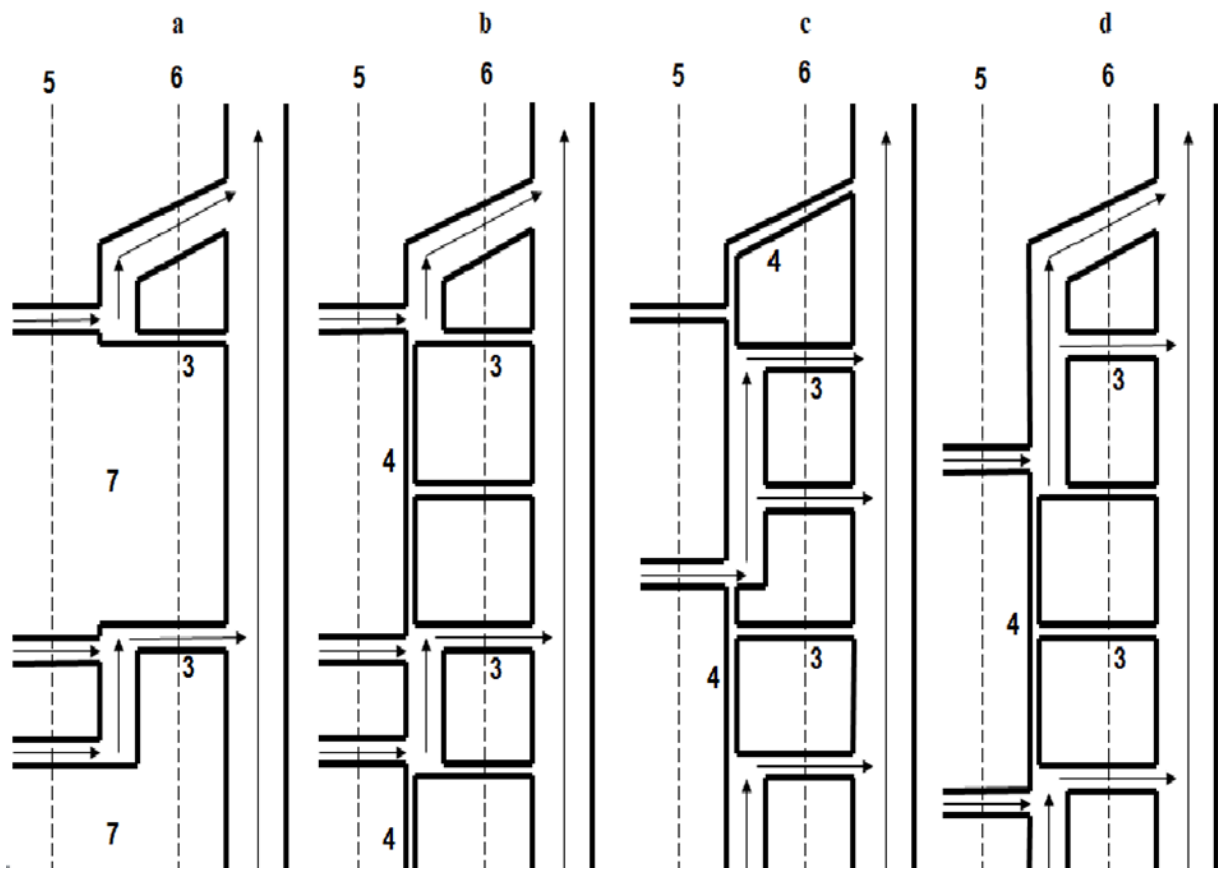

Figure 4. Schematic presentation of the variants of hypo- and/or aplastic GSV segment localization in the presence of 2 hypo- and/or aplastic segment (the arrows indicate the direction of blood flow): a) bi-segmental aplasia; b) bi-segmental hypoplasia; c) proximal segmental hypoplasia; d) distal segmental hypoplasia. 1 - deep vein; 2 - GSV; 3 - perforating veins; 4 - hypoplastic GSV region; 5 - projection of the saphenous fascia; 6 - projection of the muscular fascia; 7 - aplastic GSV region. 


\section{Variants of Localization and Extension of Hypo- and Aplasia of the Great Saphenous Vein in \\ Patients with Primary Varicose Great Saphenous Veins - 8/11}

In other $6(85.7 \%)$ cases, both underdeveloped GSV segments were hypoplastic. In 3 cases, hypoplasia, similar to aplasia, was bi-segmental (Fig. 4b) affecting the following regions of the GSV: 1) the middle and upper thirds of the tibia and the middle third of the thigh; 2) the upper third of the tibia and the middle third of the thigh; 3 ) the middle and upper thirds of the tibia and the middle and upper thirds of the thigh.

In 2 cases, the combination of segmental and distal hypoplasia (distal segmental hypoplasia) was observed (Fig. 4d). In one case, the lower third of the tibial part of the GSV and the middle third of its femoral part were affected by hypoplasia, while in the other case, hypoplasia involved the lower and middle thirds of the tibial part of the GSV and the middle third of its femoral part.

In 1 case, the combination of segmental and proximal hypoplasia was observed (proximal segmental hypoplasia) (Fig. 4c). The middle and upper thirds of the tibial part of the GSV and the upper third of its femoral part up to the terminal valve were affected by hypoplasia.

\section{Discussion}

According to the results of our study, among the patients with pathological reflux in a certain GSV segment, hypo- and aplasia of its segments were observed in $32.5 \%$ of cases $(95 \%$ CI 28.6-36.6\%). The data obtained are quite consistent with the findings of other researchers who detected hypo- and aplasia of the GSV in $12-39.8 \%$ of healthy limbs and $25 \%$ of limbs with varicose veins $[13,19,3,4,5]$. Aplasia of the GSV segments was observed twice as less frequently than hypoplasia and the difference in their detection frequencies was statistically significant. This suggested that during the ultrasound examination of the patient with pathological reflux in the GSV, hypoplasia is more likely to be detected rather than aplasia.

For a number of the results obtained, we have not found any relevant data in other research works. Firstly, we have found that the GSV trunk is not always affected by hypoplasia or aplasia only. In $2.3 \%$ of the cases (95\% CI 1.2-3.9\%), hypoplasia of a certain GSV segment evolved to aplasia, or vice versa. Secondly, we have found that the GSV trunk may have several hypo- or aplastic regions separated by its normal or varicose segment - bilevel hypo- or aplasia. Such variant of dysplasia was observed in $3.8 \%$ of the patients with hypo- or aplasia (95\% CI 1.6-7.8\%).

However, in bi-level dysplasia, there was no case of hypoplasia of one segment and aplasia of the other one: in $85.7 \%$ of the cases $(95 \%$ CI 42.1 $99.6 \%), 2$ hypoplastic regions were detected and in $14.3 \%$ of the cases (95\% CI 0.4-57.9\%), 2 aplastic regions were found.

Among the legs examined, there was no case of hypo- or aplasia of the entire GSV; although, in literature, there have been reports on such single cases, sometimes with co-existent Klippel-Trenaunay syndrome [20].

We have tried to classify the variants of hypoand aplastic GSV segment localization and extension according to the already known classifications. According to the classification of GSV aplasia described by Seidel A.C. et al. (2012), there are 6 types of GSV aplasia: Type I - aplasia only in the thigh segment; Type II - aplasia in the tibial segment; Type III - aplasia in the distal segment of the thigh and the proximal segment of the tibia; Type IV - aplasia of the whole tibial segment; Type V - aplasia of the whole tibial segment and a significant part of the thigh segment without involving a short proximal segment; Type VI - aplasia of the entire vein without involving a short segment in the tibia $[11,12]$. According to the most common classification of GSV hypoplasia described by Caggiati A., Mendoza E. (2004), there are the following types of GSV hypoplasia: A - normally sized GSV segments join the suprafascial branch; B - distal GSV segment drains into the perforating vein; $C$ - hypoplasia of the tibial part of the GSV; D - hypoplasia of the proximal part of the GSV which is shunted in the anterior accessory GSV [13].

However, both the classifications mentioned above and other well-known classifications of GVS hypo- and aplasia [9] turned out not to include all the variants we detected. We have found no systematization of bi-level hypo- and aplasia we detected, 


\section{Variants of Localization and Extension of Hypo- and Aplasia of the Great Saphenous Vein in \\ Patients with Primary Varicose Great Saphenous Veins - 9/11}

as well as the consideration of potential transition of hypoplastic GSV area into aplastic one, or vice versa. Therefore, to enable characterization of all the existing GSV hypo- and aplasia localizations, the following variants have been proposed to distinguish: 1) simple: total (along the entire trunk up to the malleolus), proximal (from the terminal valve to a certain level, but not to the malleolar level), segmental (in any GSV or SSV segments without involving its proximal and distal parts) and distal (from a certain level of the GSV or SSV trunk, but not from the terminal valve, to the medial or lateral malleolus); 2) bi-level: proximal segmental, distal segmental and bi-segmental (combinations of corresponding simple hypo- and aplasia). In addition, for every dysplastic vein segment, the type of malformation should be indicated, namely hypoplasia, aplasia, or hypo/aplasia.

There is no need for surgical removal of the GSV hypoplastic segments. However, they dramatically modify the clinical course of varicose vein disease by changing the pathways of pathological refluxes in the superficial venous system. Therefore, a thorough study of the hypo- and aplastic areas of the saphenous veins before surgery, including their mapping, is critical when planning the tactics of surgical intervention.

\section{Conclusions}

1. Among the patients with GSV reflux, the incidence rate of hypo- and aplasia of the GSV trunk was $32.5 \%$ (95\% CI 28.6-36.6\%).

2. In $2.3 \%$ of the patients with GSV reflux $(95 \%$ CI 1.2-3.9\%), hypoplasia of its certain segment evolved to aplasia, and vice versa.

3. The GSV trunk may have several hypo- or aplastic regions separated by its normal or varicose segment - bi-level hypo- or aplasia that was observed in $3.8 \%$ of the patients with hypo- or aplasia (95\% CI 1.6-7.8\%).

4. There were systematized the variants of localization and extensions of GSV hypo- and aplasia for using differential surgical tactics.

\section{Prospects of Further Researches}

Since on every lower limb we examined, in addition to recording the areas of GSV hypo- and aplasia, all the pathological venous refluxes were verified and mapped, we plan to study the relationship between different variations of pathological refluxes of the GSV and the variants of its hypo- and aplastic segment localization and extension. Such study is extremely important as it allows developing differentiated surgical tactics in different ratios between dysplastic GSV segment and segments changed by reflux.

\section{References}

[1] Uhl JF, Gillot C, Chahim M. Anatomical variations of the femoral vein. J Vasc Surg. 2010;52(3):714-719. DOI: https://doi. org/10.1016/j.jvs.2010.04.014

[PMid:20598472]

[2] Ferrara F. Embryological based identification of different anatomical patterns of superficial venous system. Acerra: Studio Flebologico Ferrara; 2013. Available from: http: //www. vasculab.it/hemodyn2013/ panel/sh/Embryological\% 20 based\%20identification\% 20 of 20 different 20 anatomical\% 20 patterns\%20of\%20superficial\% 20 venous 20 system.pdf

[3] Caggiati A, Mendoza E. Segmental hypoplasia of the great saphenous vein and varicose disease. Eur J Vasc Endovasc Surg. 2004;28(3):257-261. DOI: https://doi . org/10.1016/j.ejvs.2004.06.002 [PMid:15288628]

[4] Oguzkurt L. Ultrasonography study on the segmental aplasia of the great saphenous vein. Phlebology. 2014;29(7):447-453. DOI: https://doi.org/10.1177/ 0268355513484016 [PMid:23761865] 
[5] Caggiati A, Ricci S. The caliber of the human long saphenous vein and its congenital variations. Ann of Anat. 2000;182(2):195201. DOI: https: / / doi .org/10.1016/ S0940-9602(00) 80083-7

[6] Caggiati A, Bergan J, Gloviczki $P$ et al. Nomenclature of the veins of the lower limb: extensions, refinements, and clinical application. J Vasc Surg. 2005;41(4):719724. DOI: https: / / doi .org/10.1016/ j.jvs.2005.01.018 [PMid:15874941]

[7] Philipsen TE, De Maeseneer MG, Vandenbroeck $\mathrm{CP}$ et al. Anatomical Patterns of the Above Knee Great Saphenous Vein and its Tributaries: Implications for Endovenous Treatment Strategy. Acta Chir Belg. 2009;109(2):176179. DOI: https://doi.org/10. $1080 / 00015458.2009 .11680401$

[PMid:19499677]

[8] Choi BS, Byun GY, Hwang SB et al. Longsegment hypoplasia of great saphenous vein with posterior accessory saphenous vein as a connecting vein: a case report. Journal of Surgical Case Reports. 2017;3:1-3. DOI: https : //doi.org/10.1093/jscr/rjx058

[9] Oguzkurt L. Ultrasonography study on the segmental aplasia of the great saphenous vein. Phlebology. 2014;29(7):447-453. DOI: https://doi.org/10.1177/ 0268355513484016 [PMid:23761865]

[10] Labropoulos N, Hazelwood K, Bhatti A. Aplasia of great saphenous vein: a case report. EJVES Extra. 2006;12(6):73-75. DOI: https://doi.org/10.1016/j. ejvsextra.2006.08.001

[11] Seidel AC, Júnior PC, Rossi RM et al. Association between segmental aplasia of great saphenous vein and varicose veins of lower limbs, evaluated using color Doppler ultrasonography. J Vasc Bras. 2015;14(3):211-
216. DOI: https://doi.org/10.1590/ $1677-5449.0315$

[12] Seidel AC, Cavalari Jr P, Rossi RM et al. Proposal for classification of the great saphenous vein aplasia by the echo-color Doppler. VEITH Symposium. 2014; New York. Tema livre com apresentação oral.

[13] Caggiati A, Mendoza T. Segmental Hypoplasia of the Great Saphenous Vein and Varicose Disease. Eur J Vasc Endovasc Surg. 2004;28(3):257-261. DOI: https://doi . org/10.1016/j.ejvs.2004.06.002 [PMid:15288628]

[14] Oguzkurt L. Ultrasonographic anatomy of the lower extremity superficial veins. Diagn Interv Radiol 2012;18:423-430. DOI: https://doi.org/10.4261/ 1305-3825.DIR.5321-11.1

[15] Eklöf B, Rutherford RB, Bergan JJ et al. Revision of the CEAP classification for chronic venous disorders: Consensus statement. Journal of Vascular Surgery. 2004;40(6):1248-1252. DOI: https : / / doi . org/10.1016/j.jvs.2004.09.027 [PMid:15622385]

[16] Coleridge-Smith P, Labropoulos N, Partsch $\mathrm{H}$ et al. Duplex Ultrasound Investigation of the Veins in Chronic Venous Disease of the Lower Limbs - UIP Consensus Document. Part I: Basic Principles. Eur J Vasc Endovasc Surg. 2006;31(1):8392. DOI: https://doi.org/10.1016/ j.ejvs.2005.07.019 [PMid:16226898]

[17] Cavezzi A, Labropoulos N, Partsch H et al. Duplex Ultrasound Investigation of the Veins in Chronic Venous Disease of the Lower Limbs - UIP Consensus Document. Part II. Anatomy. Eur J Vasc Endovasc Surg. 2006;31(3):288299. DOI: https://doi .org/10.1016/ j.ejvs.2005.07.020 [PMid:16230038] 
[18] R Development Core Team. R: A language and environment for statistical computing. R Foundation for Statistical Computing. 2013. Available from: http://www . R-project.org/

[19] Caggiati A, Ricci S. The caliber of the human long saphenous vein and its congenital variations. Analis of Anatomy. 2000;182(2):195201. DOI: https://doi.org/10.1016/ S0940-9602(00) 80083-7

[20] Herman J, Musil D. Klippel-Trénaunay syndrome associated with great saphenous vein aplasia. Phlebology. 2010;25(1):35-37. DOI: https://doi.org/10.1258/ phleb.2009.008079 [PMid:20118344]

Received: 2019-12-11

Revised: 2019-12-19

Accepted: 2019-12-19 